\title{
Analysis of Post-operative Adjuvant Chemotherapy Versus Adjuvant Chemoradiation Therapy Outcomes in Non-metastatic Cholangiocarcinoma: an NCDB Review
}

\author{
Robin R. Rodriguez ${ }^{1}$ (1) $\cdot$ Stephen Abel ${ }^{2} \cdot$ Jyothika Mamadgi $^{1} \cdot$ Paul B. Renz ${ }^{2} \cdot$ Rodney E. Wegner $^{2} \cdot$ Moses S. Raj $^{1}$
}

Accepted: 19 August 2021 / Published online: 6 September 2021

(c) The Author(s) 2021

\begin{abstract}
Background Each year, approximately 8000 cases of cholangiocarcinoma are recorded in the USA. Surgical resection is considered to be the only curative option. Despite surgery as a curative approach, many patients will require adjuvant therapies in the form of chemotherapy (ChT) or chemoradiotherapy (CRT). As such, we sought to analyze outcomes in patients with non-metastatic cholangiocarcinoma receiving adjuvant ChT or CRT following surgical resection.

Methods We queried the National Cancer Database (NCDB) for patients with a diagnosis of non-metastatic cholangiocarcinoma between the years 2010 and 2015 who underwent adjuvant ChT or CRT following surgery. Overall survival (OS) was calculated using Kaplan Meier method. Cox proportional hazard ratios were used to identify predictors of overall survival, and logistic regression was used to identify predictors of receiving each treatment.

Results A total of 875 patients were identified who met the above eligibility criteria. Of these patients, 818 received adjuvant chemotherapy alone with 57 patients receiving adjuvant chemoradiation therapy. The median OS in patients receiving CRT was 19.8 months versus 11.9 months for ChT ( $p$ value $<0.0238$ ). The 1 - and 5 -year survival rates between ChT and CRT were $50 \%$ vs $61 \%$ and $6 \%$ vs $13 \%$, respectively (hazard ratio $0.7005 ; 95 \%$ CI $0.51-0.97$; $p$ value $<0.0294$ ).

Conclusion The results of this study suggest a potential benefit of chemoradiation therapy in the adjuvant setting, although the trends appear to show rare utilization. Given the limitations of our study, prospective corroboration is warranted.
\end{abstract}

Keywords Cholangiocarcinoma $\cdot$ Adjuvant treatment $\cdot$ Chemotherapy $\cdot$ Radiation therapy

\section{Introduction}

With an incidence of approximately 8000 cases annually in the USA, cholangiocarcinoma is a rare form of cancer [1]. Recent studies are beginning to demonstrate the complex nature of this disease, with important implications stemming from its molecular pathogenesis [2]. This heterogeneous disease derives from the epithelium and is categorized according to its anatomic location as either intrahepatic or extrahepatic [2,3]. Each of these types presents unique challenges in treatment and management due to their different

Robin R. Rodriguez

robin.rodriguez@ahn.org

1 Department of Medical Oncology, Allegheny Health Network Cancer Institute, Allegheny General Hospital, Level 01, 320 E. North Avenue, Pittsburgh, PA 15212, USA

2 Department of Radiation Oncology, Allegheny Health Network, Cancer Institute Pittsburgh, Pa, USA epidemiology and prognosis [4]. At present, surgical resection is the only potential curative option [3]. This option however is not available for many patients considering that the disease is either locally advanced or metastatic at diagnosis [5]. Even with curative resection, many patients require adjuvant therapies in the form of chemotherapy (ChT) alone or chemoradiotherapy (CRT) [6]. Due to the risk of recurrence, it is recommended that patients are either enrolled in clinical trials or begun on adjuvant therapy [6]. To indicate the rationale for the use of adjuvant therapy in post-operative patients, a phase III clinical trial demonstrated an improvement in overall survival in patients receiving adjuvant chemotherapy versus surgery alone [7]. Benefits reported with the use of adjuvant chemoradiation have been sparse, with many studies being interpreted with mixed results [8]. As such, we sought to analyze survival outcomes and identify variables predictive of adjuvant ChT or CRT receipt in patients with non-metastatic cholangiocarcinoma using the National Cancer Database (NCDB). 


\section{Methods}

The NCDB is a joint program which is managed by both the American Cancer Society and the American College of Surgeons [9]. This oncological database represents approximately $70 \%$ of cancer cases in the USA annually and extrapolates its data from over 1500 Commission on Cancer (CoC)-accredited facilities [9]. As the information contained within the database is de-identified, this study was exempt from institutional board review (IRB) supervision. The results and analysis included herein have not been verified by either the American Cancer Society or the American College of Surgeons; and these programs do not take responsibility for the conclusions that result from this study. As this methodology has been undertaken in previous studies, a similar analysis has been done to extrapolate these results [10, 11].
Within this study, we utilized the NCDB liver database from 2010 to 2015. Cholangiocarcinoma, adenocarcinoma, and Klatskin tumor of histology codes 8140/8160/8162, respectively, were used [12, 13]. Inclusion criteria were all cases that were clinically and pathologically non-metastatic and those which received surgery as their first treatment option. Patients who did not receive either postoperative chemotherapy or chemoradiation therapy were excluded along with those patients who had less than 2 months follow-up to account for immortal time bias. A CONSORT diagram that depicts the inclusion criteria is shown in Fig. 1. Utilizing the exclusion criteria, a total of 875 patients were eligible for final analysis. Of these 875 patients, 818 were found to have received chemotherapy alone, whilst 57 patients received both chemotherapy and radiation therapy. The information that was analyzed from the database on the patients included clinical, treatment, and

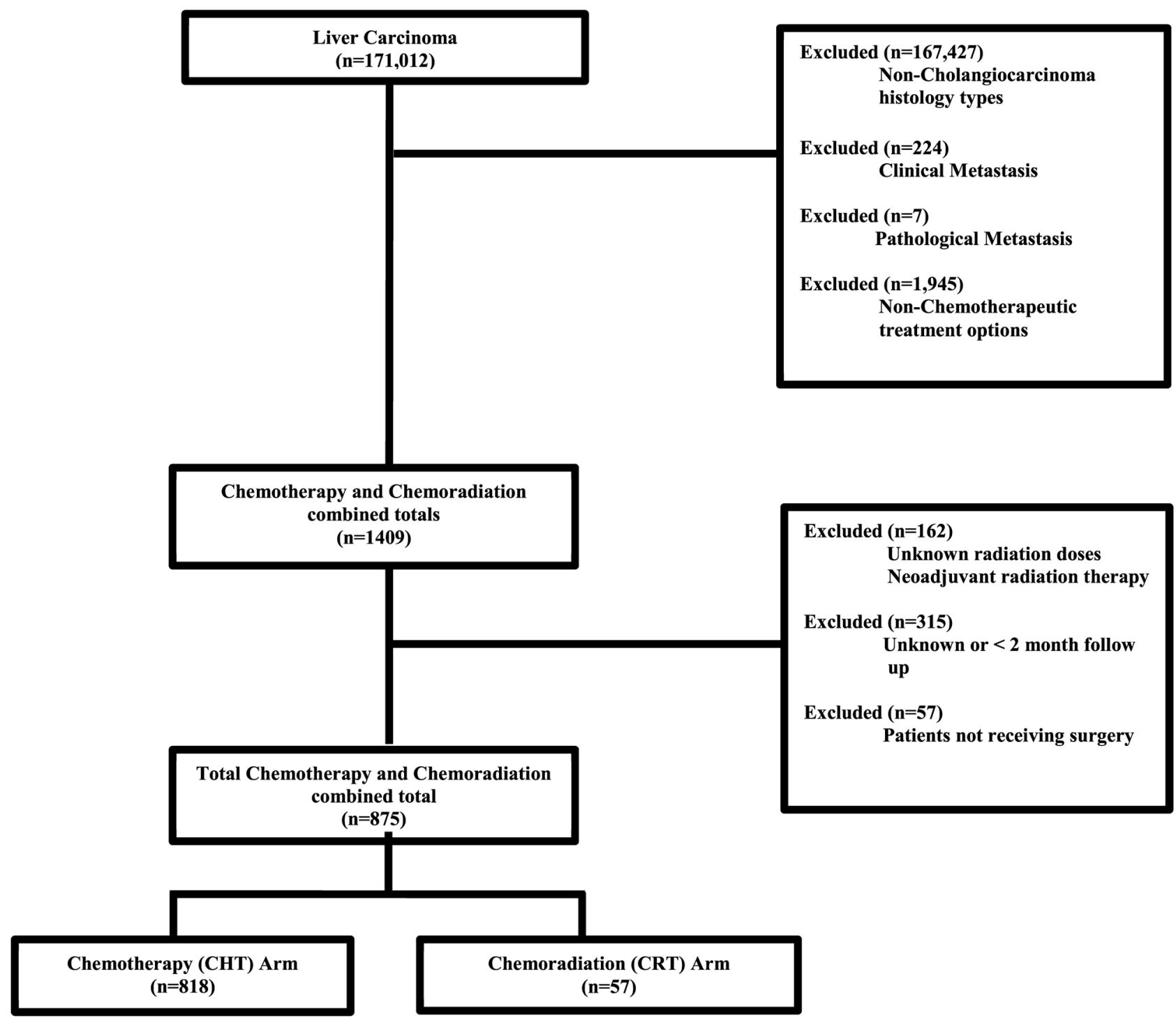

Fig. 1 CONSORT Diagram. Chemotherapy vs chemoradiation therapy 
baseline characteristics. Race was defined as either white, African American, or other/unknown. Data was obtained from this information by the performance of statistical analysis via MedCalc Version 18 (Ostend, Belgium).

The results were reported from the use of both univariable and multivariable logistic regression models used to determine associated parameters of interest. The overall survival was calculated using the date of diagnosis and the date of last contact or time of death with a Kaplan-Meier curve. Univariable survival analysis was carried out for all characteristics as listed in Table 1. Following, statistically significant parameters were then used to determine adjusted hazard ratios (HR) along with a 95\% confidence interval (CI) calculated with a $p$ value of 0.05 . A Cox proportional hazards model was developed to assess relation of multivariable parameters.

\section{Results}

From the NCDB database between the years of 2010 and 2015 , a total of 875 patients with non-metastatic cholangiocarcinoma treated with either adjuvant chemotherapy or adjuvant chemoradiotherapy following surgery were eligible
Table 1 Patient and treatment characteristics $(N=15,110)$

\begin{tabular}{|c|c|c|c|}
\hline Characteristic & No. (\% or range) & Characteristic & No. (\% or range) \\
\hline \multirow[t]{2}{*}{ Demographics } & & Treatment facility type & \\
\hline & & Community cancer program & $483(3.2)$ \\
\hline Sex & & Academic/research program & $6570(43.5)$ \\
\hline Male & $6892(46)$ & Comprehensive cancer program/other & $8057(53.3)$ \\
\hline Female & $8218(54)$ & Rural counties & $339(2.3)$ \\
\hline Age & & Year of treatment & \\
\hline Median & $75(40-90)$ & 2004-2007 & $526(3.5)$ \\
\hline$\leq 65$ & $2424(16.0)$ & 2008-2011 & $4252(28.1)$ \\
\hline$>65$ & $12,686(84.0)$ & $2012-2015$ & $10,332(68.4)$ \\
\hline Race & & Disease characteristics & \\
\hline White & $13,617(90.1)$ & & \\
\hline African American & $1190(7.9)$ & Clinical T stage & \\
\hline Other/unknown & $303(2.0)$ & $\mathrm{T} 1$ & $11,845(78.4)$ \\
\hline Comorbidity score & & $\mathrm{T} 2$ & $3,265(21.6)$ \\
\hline 0 & $8558(56.6)$ & Histology & \\
\hline 1 & $4031(26.7)$ & Adenocarcinoma & $8924(59.1)$ \\
\hline $2+$ & $2521(16.7)$ & Squamous Cell Carcinoma & $6186(40.9)$ \\
\hline Insurance & & Grade & \\
\hline Private & $1869(12.4)$ & Well differentiated & $1453(9.6)$ \\
\hline Government & $12,951(85.7)$ & Moderately differentiated & $3033(20.1)$ \\
\hline Unknown & $180(1.2)$ & Poorly differentiated & $2833(18.8)$ \\
\hline Education & & Unknown & $7791(51.6)$ \\
\hline$\geq 29$ & $2113(14.0)$ & Treatment characteristics & \\
\hline 20 to 28.9 & $4138(27.4)$ & Radiation dose, Gy & \\
\hline 14 to 19.9 & $5378(35.6)$ & Median (range) & \\
\hline$<14$ & $3435(22.7)$ & & $50.0(30.0-75.0)$ \\
\hline Unknown & $54(0.3)$ & Interquartile range (Gy) & \\
\hline Income, US dollars & & & 5.5 \\
\hline$<30,000$ & $2685(17.8)$ & Fractionation & \\
\hline 30,000 to 35,000 & $3890(25.7)$ & Median (fraction number) & \\
\hline 35,000 to 45,999 & $4343(28.7)$ & & $4(1-5)$ \\
\hline$\geq 46,000$ & $4132(27.4)$ & Biologically equivalent dose, Gy10 & \\
\hline Unknown & $60(0.4)$ & Median (Range) & \\
\hline $\begin{array}{l}\text { Distance to treatment } \\
\text { facility, miles }\end{array}$ & & & $112.5(100-231.9)$ \\
\hline$\leq 10$ & $7046(46.6)$ & Interquartile Range (Gy10) & \\
\hline$>10$ & $8064(53.3)$ & & 51.2 \\
\hline
\end{tabular}


Table 2 Comparative analysis of adjuvant chemotherapy versus adjuvant chemoradiation therapy by baseline characteristics in non-metastatic cholangiocarcinoma cases

\begin{tabular}{|c|c|c|c|c|c|}
\hline Characteristic & $\begin{array}{l}\text { Chemother- } \\
\text { apy (ChT) } \\
(n=818) \\
(\%)\end{array}$ & $\begin{array}{l}\text { Chemoradiation } \\
\text { therapy }(\mathrm{CRT})(n=57) \\
(\%)\end{array}$ & Odds ratio & $95 \% \mathrm{CI}$ & $p$ \\
\hline \multicolumn{6}{|l|}{ Sex } \\
\hline Male & 395 (48) & $32(56)$ & 1 & Ref & \\
\hline Female & $423(52)$ & $25(44)$ & 0.80 & $0.44-1.47$ & 0.46 \\
\hline \multicolumn{6}{|l|}{ Race } \\
\hline White & $708(87)$ & $53(94)$ & 1 & Ref & \\
\hline African American & $62(7)$ & $2(3)$ & 0.69 & $0.15-3.14$ & 0.63 \\
\hline Other & $48(5)$ & $2(3)$ & 0.83 & $0.18-3.82$ & 0.81 \\
\hline \multicolumn{6}{|l|}{ Comorbidity score } \\
\hline 0 & $557(68)$ & $43(75)$ & 1 & Ref & \\
\hline 1 & $184(23)$ & $12(21)$ & 0.79 & $0.38-1.64$ & 0.52 \\
\hline$\geq 2$ & $77(9)$ & $2(4)$ & 0.34 & $0.08-1.49$ & 0.15 \\
\hline \multicolumn{6}{|l|}{ Age } \\
\hline$\geq 65$ & $435(53)$ & $28(49)$ & 1 & Ref & \\
\hline$<65$ & $383(47)$ & $29(51)$ & 1.028 & $0.50-2.13$ & 0.94 \\
\hline \multicolumn{6}{|l|}{ Insurance } \\
\hline None & $25(3)$ & $1(1)$ & 1 & Ref & \\
\hline Private payer & $326(40)$ & $29(52)$ & 2.76 & $0.31-24.51$ & 0.36 \\
\hline $\begin{array}{l}\text { Government } \\
\text { Unknown }\end{array}$ & $\begin{array}{l}458(56) \\
9(1)\end{array}$ & $\begin{array}{l}26(46) \\
1(1)\end{array}$ & $\begin{array}{l}1.63 \\
4.76\end{array}$ & $\begin{array}{l}0.18-14.45 \\
0.22-102.29\end{array}$ & $\begin{array}{l}0.66 \\
0.32\end{array}$ \\
\hline \multicolumn{6}{|l|}{ Facility type } \\
\hline $\begin{array}{l}\text { Community Cancer } \\
\text { Program/Comprehensive } \\
\text { Cancer }\end{array}$ & $419(51)$ & $21(37)$ & 1 & Ref & \\
\hline Academic Research & $315(39)$ & $29(51)$ & 1.32 & $0.67-2.58$ & 0.42 \\
\hline Integrated Cancer Network & $84(10)$ & $7(12)$ & 2.05 & $0.77-5.46$ & 0.15 \\
\hline \multicolumn{6}{|l|}{ Income, USD } \\
\hline$<30,000$ & $133(16)$ & $12(21)$ & 1 & Ref & \\
\hline $30,000-35,000$ & $216(26)$ & $9(16)$ & 0.36 & $0.13-1.04$ & 0.06 \\
\hline $35,000-45,999$ & $228(28)$ & $15(26)$ & 0.65 & $0.23-1.80$ & 0.40 \\
\hline$>46,000$ & $241(30)$ & $21(37)$ & 0.70 & $0.23-2.16$ & 0.53 \\
\hline \multicolumn{6}{|l|}{ Education } \\
\hline$\geq 29 \%$ & $146(18)$ & $9(16)$ & 1 & Ref & \\
\hline 20 to 28.9 & $221(27)$ & $15(26)$ & 1.06 & $0.40-2.81$ & 0.90 \\
\hline 14 to 19.9 & $264(32)$ & $18(32)$ & 1.22 & $0.41-3.61$ & 0.72 \\
\hline$<14$ & $187(23)$ & $15(26)$ & 1.24 & $0.37-4.18$ & 0.73 \\
\hline \multicolumn{6}{|l|}{ T stage } \\
\hline 1 & $44(5)$ & $6(10)$ & 1 & Ref & \\
\hline 2 & $40(5)$ & $9(16)$ & 1.77 & $0.49-6.44$ & 0.39 \\
\hline 3 & $20(2)$ & $0(0)$ & $2.32^{\mathrm{E} 009}$ & $0.31-16.04$ & 1.00 \\
\hline 4 & $9(1)$ & $2(4)$ & 2.23 & $0.08-1.71$ & 0.42 \\
\hline Unknown & $705(87)$ & $40(70)$ & 0.37 & & 0.20 \\
\hline \multicolumn{6}{|l|}{ Surgical margins } \\
\hline No & $60(7)$ & $9(16)$ & 1 & Ref & \\
\hline Yes & $13(2)$ & $5(9)$ & 6.38 & $1.46-27.85$ & $<0.01$ \\
\hline Unknown & $745(91)$ & $43(75)$ & 0.63 & $0.25-1.55$ & 0.31 \\
\hline \multicolumn{6}{|l|}{ Distance to facility } \\
\hline$\geq 11$ miles & $383(47)$ & $38(67)$ & 1 & Ref & \\
\hline$<11$ miles & $435(53)$ & $19(33)$ & 0.84 & $0.78-0.89$ & $<0.02$ \\
\hline
\end{tabular}

Education is quartiles of the percentage of persons with less than a high school education in the patients' residence census tract. Income is median household income in the patients' residence census tract 
for assessment. From these patients, the baseline characteristics are demonstrated in Table 1. Median age was 62 years. The majority of patients were white in race $(87 \%)$, and there was a small predominance of female over male patients ( $51 \%$ and $49 \%$, respectively). Although the data collected was from 2010 to 2015 , no cases of cholangiocarcinoma were able to be included from 2015 due to our exclusion criteria. Thus, only cases from the years 2010-2014 were included in this study. The majority of the cases $(66 \%)$ took place between 2012 and 2014. Table 2 details differences within demographic and disease-related characteristics between those who received chemotherapy versus chemoradiotherapy. Statistical significance was determined for two variables. Patients were more likely to obtain chemoradiation if they had positive surgical margins $(p<0.01)$. In addition, patients receiving chemoradiation were more likely to live closer to the treatment facility $(p<0.02)$. Using Kaplan Meier analysis, the median overall survival was calculated for both cohorts. Median OS was 11.9 months for ChT and 19.8 months for CRT. At 1 year, 3 years, and 5 years, the OS for ChT was $50 \%, 16 \%$, and $6 \%$ versus CRT at $61 \%, 22 \%$, and $13 \%$, respectively $(p<0.02)$. These results can be seen in Fig. 2. Median follow-up collectively for all cases was determined to be 11.4 months. The interquartile range for follow-up was 5.7-21.4. On multivariable analysis, it was determined that improved OS was associated with CRT, female gender, lower co-morbidity score, and race other than white or African American (Table 3).

\section{Discussion}

Cholangiocarcinoma, a rare biliary tract cancer, is a heterogeneous disease with an aggressive natural history [2]. In addition to anatomic location, surgical margins are known prognostic factors with $\mathrm{R} 0$ resected margins being shown to have clinically better outcomes and improved 5-year OS [14, 15]. Despite surgical resection being a potentially curative option, a very limited number of patients with cholangiocarcinoma are eligible for surgical resection [4]. Additionally, those with surgical resection still have the potential for recurrence. A retrospective study involving patients with intrahepatic cholangiocarcinoma demonstrated 1-year, 2-year, and 3-year recurrence-free rates of only $16.2 \%, 5.4 \%$, and $2.7 \%$, respectively [16]. Corroborating this, a large case series of 920 patients treated with surgical resection for intrahepatic cholangiocarcinoma demonstrated 607 patients (66\%) who developed recurrence of the disease [17]. Comparatively, in surgically resected perihilar and distal extrahepatic cholangiocarcinoma, the recurrence rate ranged from 60 to 75\% [18]. Poor prognosis of this disease and the risk of recurrence following surgical resection in local stages illustrate the imperative need to consider adjuvant options for management. Chemotherapy and chemoradiation therapy are two of the main adjuvant options offered to non-metastatic cholangiocarcinoma patients following surgical resection. Current clinical trials studying the effects of these options include

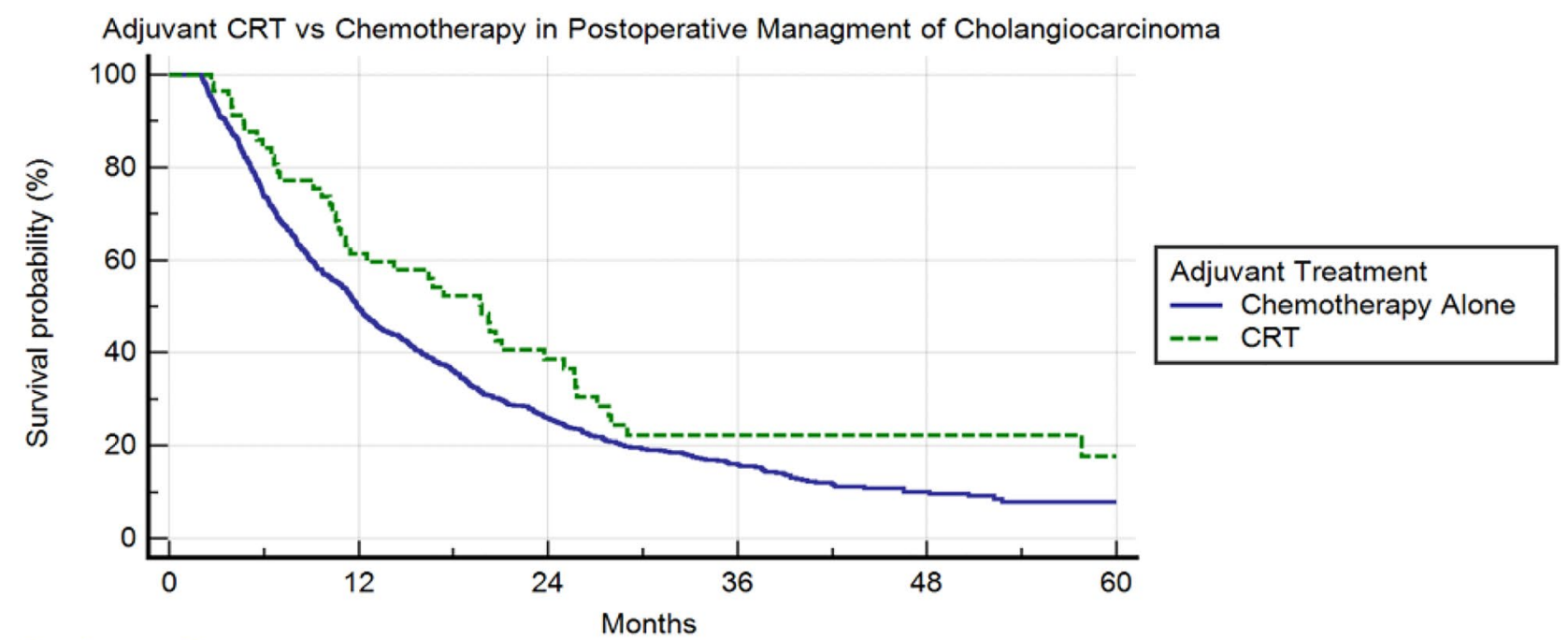

Number at risk
Group: Chemotherapy
$\begin{array}{cc}818 & 3 l o n e \\ \text { Group: CRT } & 377 \\ 57 & 35\end{array}$

Fig. 2 Kaplan Meier analysis 
Table 3 Multivariable Cox proportional hazards models for overall survival in patients with resected non-metastatic cholangiocarcinoma receiving adjuvant chemotherapy versus chemoradiotherapy

\begin{tabular}{|c|c|c|}
\hline Significant characteristic & Hazard of death (95\% CI) & $p$ \\
\hline & Cox model & \\
\hline \multicolumn{3}{|l|}{ Age } \\
\hline$\geq 65$ & Reference & \\
\hline$<65$ & $1.02(0.84-1.23)$ & 0.86 \\
\hline \multicolumn{3}{|l|}{ Sex } \\
\hline Male & Reference & \\
\hline Female & $0.79(0.68-0.92)$ & $<0.003$ \\
\hline \multicolumn{3}{|l|}{ Insurance } \\
\hline None & Reference & \\
\hline Government & $1.16(0.72-1.88)$ & 0.53 \\
\hline Private & $1.00(0.62-1.60)$ & 0.99 \\
\hline Unknown & $1.07(0.44-2.60)$ & 0.89 \\
\hline \multicolumn{3}{|l|}{ Comorbidity score } \\
\hline 0 & Reference & \\
\hline 1 & $0.92(0.76-1.11)$ & 0.37 \\
\hline$\geq 2$ & $1.40(1.07-1.83)$ & $<0.01$ \\
\hline \multicolumn{3}{|l|}{ Race } \\
\hline White & Reference & \\
\hline African American & $1.07(0.79-1.43)$ & 0.67 \\
\hline Other & $0.61(0.42-0.88)$ & $<0.008$ \\
\hline \multicolumn{3}{|l|}{ Income } \\
\hline$<38,000$ & Reference & \\
\hline $38,000-47,999$ & $1.09(0.84-1.42)$ & 0.51 \\
\hline $48,000-62,999$ & $0.97(0.73-1.28)$ & 0.82 \\
\hline$\geq 63,000$ & $0.94(0.70-1.60)$ & 0.71 \\
\hline \multicolumn{3}{|l|}{ Facility type } \\
\hline $\begin{array}{l}\text { Community Cancer Program/ } \\
\text { Comprehensive }\end{array}$ & Reference & \\
\hline Academic/Research Program & $0.95(0.80-1.13)$ & 0.56 \\
\hline Integrated Network Cancer & $0.87(0.66-1.12)$ & 0.28 \\
\hline \multicolumn{3}{|l|}{ Education } \\
\hline$\geq 29 \%$ & Reference & \\
\hline 20 to 28.9 & $1.15(0.89-1.48)$ & 0.27 \\
\hline 14 to 19.9 & $1.12(0.85-1.47)$ & 0.43 \\
\hline$<14$ & $1.30(0.95-1.77)$ & 0.10 \\
\hline \multicolumn{3}{|l|}{ Radiation treatment } \\
\hline Chemotherapy & Reference & \\
\hline Chemoradiotherapy & $0.70(0.51-0.97)$ & $<0.03$ \\
\hline
\end{tabular}

Education is quartiles of the percentage of persons with less than a high school education in the patients' residence census tract. Income is median household income in the patients' residence census tract

the Adjuvant Chemotherapy with Gemcitabine and Cisplatin Compared to Standard of Care After Curative Intent Resection of Biliary Tract Cancer (ACTICCA-1 trial) [19]. The results of this trial are currently still pending [19]. Continued studies such as ACTICCA-1 were initiated due to the encouraging findings of the BILCAP (capecitabine compared with observation in resected biliary tract cancer) trial [20]. This phase III randomized, multicenter study analyzed 447 patients, 223 of which were given capecitabine following surgical resection [20]. The results found that the recurrence-free survival for the capecitabine group was 24.4 months (95\% CI, 19.8-46.3) versus 17.5 months in the control group (95\% CI, 12.0-23.8) [20]. Aside from capecitabine, the European Study Group for Pancreatic Cancer (ESPAC-3) trial also demonstrated efficacy of both gemcitabine and fluorouracil with a median OS of 43.1 months (95\% CI, 34.0-56.0) for the two chemotherapy groups versus 35.2 months (95\% CI, 27.2-43.0) for the control group [21]. Although the ESPAC-3 focused on periampullary cancers, it is understood that cholangiocarcinoma cases are often included in studies of periampullary disease [6]. Another phase III trial conducted by investigators in the UK assessing the role of chemotherapy in biliary tract cancers also provided insight into multi versus single agent therapy use [22]. Within this study, 410 patients with locally advanced or metastatic cholangiocarcinoma and other biliary tract cancers were assessed [22]. The cohort arms were divided into either cisplatin followed by gemcitabine vs gemcitabine alone [22]. The study identified an increased median OS and progressionfree survival in the combination chemotherapy group vs the single-agent therapy group (11.7 months/8 months vs 8.1 months/5 months) [22]. The aforementioned study largely lends to the importance of considering multi agent therapies and corroborates the efficacious findings of chemotherapy in management of cholangiocarcinoma.

The use of chemoradiation therapy in the adjuvant setting, unlike chemotherapy alone, has demonstrated more heterogeneous results. Of note, chemoradiation therapy is often used in adjuvant management of patients with positive R1 resection margins [6]. This is similar to findings within our study that CRT was utilized more in patients with positive margins. Illustrating this, one retrospective review studied patients with $\mathrm{R} 0$ margins treated with surgery-only versus $\mathrm{R} 1$ margin patients treated with adjuvant CRT post-operatively [8]. The results demonstrated similar OS between the two groups ( $42 \%$ versus $36 \%, p<0.6$ ) [8]. On the other hand, contrary to the aforementioned findings, a phase III trial conducted by the European Organization of Cancer Research (EORTC) did not demonstrate oncologic benefit with the addition of adjuvant chemoradiation [23]. A limitation of this study was that the cholangiocarcinoma arm was relatively small. [23].

In comparing differing adjuvant therapies and their effect on survival, previous research has been conducted. One such study reviewed outcomes of 599 patients who were administered adjuvant therapy following surgical resection in intrahepatic cholangiocarcinoma cases [24]. These results illustrated improved survival with CRT versus ChT 
alone [24]. Overall, the results of our study stand to corroborate the findings of Lin et al. in that, patient receiving CRT had improved OS versus ChT alone [24]. Within the trial of Lin et al., the 2-year OS between concurrent chemoradiotherapy and chemotherapy alone was $48 \%$ versus $38 \%$ [24]. In comparison of these findings in conjunction with our study, the results for overall survival are similar. It is important to note that although 3-5-year survival data is often of particular interest, both our study and that of Lin et al., the 1- and 2-year survival OS was significant to address as the median OS of cholangiocarcinoma in and of itself is relatively short [24]. A limitation of our study in this regard however is the lack of separation between concurrent and sequential radiotherapy. Another study assessed the NCDB database for the results of differing adjuvant therapies [25]. This study looked at patients from the years 1998-2006 who were treated for extrahepatic cholangiocarcinoma [25]. This study by Hoehn et al. had a large sample population with a total of 8741 patients [25]. Within their study, 3 arms where identified: surgery alone, adjuvant ChT, or CRT following surgery [25]. From these groups, the patients given adjuvant CRT were noted to have increased OS versus those who had surgery only or adjuvant ChT [25]. Additionally, and similar to our study, Hoehn et al. also concluded that females were more likely to have better outcomes than males [25]. Other important results from their study were the corroboration that worse survival was associated with positive margins along with advanced stage of disease [25]. Another NCDB review of extrahepatic cholangiocarcinoma from the years 2004-2014 also noted increased survival with CRT adjuvant therapy [26]. However, this increased survival with the use of CRT versus ChT was only noted in patients with positive resection margins only [26]. Without the positive margins, OS was similar between the ChT and CRT groups at a total of 36 months [26]. Although the aforementioned study along with others demonstrated the significance of surgical margins in relation to OS, our study was not able to corroborate that margins were predictive of survival due in part to our small sample size $[25,26]$. In regard to studies comparing adjuvant therapies in intrahepatic cholangiocarcinoma, a study done of the NCDB from the years 1998-2006 revealed that there was increased survival in patients with positive resection margins who received either type of adjuvant therapy (CRT or ChT) [27]. In this study, it was concluded that there was no significant improvement in survival with adjuvant therapy if the patients were node negative [27]. In this study, analysis of ChT versus CRT was not conducted; so, no conclusions can be drawn as to whether one type of adjuvant therapy conferred increased survival benefit over the other [27].

Ultimately, the results of our study provide further evidence for the need of continued clinical trials to validate the use of adjuvant therapies in patients with cholangiocarcinoma. Although multiple studies have been undertaken to assess the benefit of adjuvant therapy in post-resected cholangiocarcinoma cases, there is still no consensus as to which type of adjuvant therapy (CRT or ChT) is more beneficial in relation to overall survival. Thus, further research should be directed towards determining OS between chemoradiation therapy versus chemotherapy alone as mounting evidence inclusive of the results of our study are illustrating that CRT versus ChT alone is associated with improved OS. Although our study is not the first to be done in reporting results of cholangiocarcinoma cases, the use of the NCDB offers advantages over single institution cohorts alone. Consequently, the information contained therein can be applied to a greater population of cholangiocarcinoma patients. Additionally, our report is one of a few studies that focuses solely on nonmetastatic cases. This is important in that it is evident that even in local stages, adjuvant therapy needs to be a consideration for continued management of this disease due to the potential for recurrence as previously mentioned [6, 16]. Despite the aforementioned results of CRT associated with improved OS, it is important to note that our study is not without limitations. These limitations are inclusive of selection bias as the patient data was obtained via NCDB, inability to distinguish intrahepatic versus extrahepatic disease, inability to report patient functional status asides from comorbidity score, along with unknown rates of local or distant control. Additionally, despite statistically significant findings in relation to surgical margins, the majority of margins were unknown as well as the majority of $\mathrm{T}$ staging. Other limitations revolve around unknown variables surrounding chemotherapy and chemoradiotherapy such as unknown chemotherapy agents, unknown duration, unknown toxicity profile, and unknown number of cycles. Our criterion was also limited to non-metastatic cases. Lastly and most potentially the largest limitation of our study is the relatively small sample size of our chemoradiation group. Consequently, retrospective studies should be further corroborated by phase III trials which can properly randomize and diminish confounding variables.

\section{Conclusion}

Overall, the scope of our study was to review adjuvant treatment options and their outcomes for post-operative patients with non-metastatic cholangiocarcinoma. The results suggest a potential benefit of chemoradiation therapy in the adjuvant setting over chemotherapy alone; although the trends appear to show rare utilization. Given the limitations of our study, prospective corroboration is warranted.

This study has not been presented or published in part or full in any other form elsewhere. 
Acknowledgements The authors would like to extend our appreciation to the Department of Medical Oncology and the Department of Radiation Oncology in Allegheny Health Network for their assistance in allowing us to continue pursuing knowledge within our respected fields.

Author Contribution (I) Conception and design: All authors. (II) Administrative support: None. (III) Provision of study materials or patients: None. (IV) Collection and assembly of data: RR Rodriguez, S Abel, J Mamadgi. (V) Data analysis and interpretation: RR Rodriguez, S Abel. (VI) Manuscript writing: All authors. (VII) Final approval of manuscript: All authors.

Availability of Data and Material The information that was used to produce the resulting graphs and statistical analysis was derived from the National Cancer Database (NCDB).

Code Availability Not applicable.

\section{Declarations}

Ethical Statement The authors are accountable for all aspects of the work in ensuring that questions related to the accuracy or integrity of any part of the work are appropriately investigated and resolved.

Consent to Participate and for Publication The data contained via the use of the NCDB is de-identified and therefore no patient records were obtained for the use in this project.

Conflicts of Interest The authors declare no conflict of interest. All authors have completed the ICMJE uniform disclosure form.

Checklist The authors have completed the STROBE reporting checklist.

Open Access This article is licensed under a Creative Commons Attribution 4.0 International License, which permits use, sharing, adaptation, distribution and reproduction in any medium or format, as long as you give appropriate credit to the original author(s) and the source, provide a link to the Creative Commons licence, and indicate if changes were made. The images or other third party material in this article are included in the article's Creative Commons licence, unless indicated otherwise in a credit line to the material. If material is not included in the article's Creative Commons licence and your intended use is not permitted by statutory regulation or exceeds the permitted use, you will need to obtain permission directly from the copyright holder. To view a copy of this licence, visit http://creativecommons.org/licenses/by/4.0/.

\section{References}

1. American Cancer Society [Internet]. Key statistics for bile duct cancer; 2018 Jul 3 [cited 2021 Feb 1]. Available from: https:// www.cancer.org/cancer/bile-duct-cancer/about/key-statistics.html

2. Labib PL, Goodchild G, Pereira SP. Molecular pathogenesis of cholangiocarcinoma. BMC Cancer. 2019;19(1):185.

3. Lowe R, Anderson C. UpToDate. Epidemiology, pathogenesis, and classification of cholangiocarcinoma; 2020 Nov 10 [cited 2021 Feb 1].

4. Rizvi S, Khan SA, Hallemeier CL, et al. Cholangiocarcinomaevolving concepts and therapeutic strategies. Nat Rev Clin Oncol. 2018;15(2):95-111.

5. Cillo U, Fondevilla C, Donadon M, et al. Surgery for cholangiocarcinoma. Wiley Liver Int. 2019;39(Suppl 1):143-55.
6. Anderson C, Stuart K. UpToDate. Treatment of localized cholangiocarcinoma: Adjuvant and neoadjuvant therapy and prognosis; 2021 [cited 2021 Feb 1].

7. Takada T, Amano H, Yasuda H, Nimura Y, Matsushiro T, Kato $\mathrm{H}$, et al. Is postoperative adjuvant chemotherapy useful for gallbladder carcinoma? A phase III multicenter prospective randomized controlled trial in patients with resected pancreaticobiliary carcinoma. Cancer. 2002;95(8):1685-95.

8. Borghero Y, Crane CH, Szklaruk J, Oyarzo M, Curley S, Pisters PW, et al. Extrahepatic bile duct adenocarcinoma: patients at high-risk for local recurrence treated with surgery and adjuvant chemoradiation have an equivalent overall survival to patients with standard-risk treated with surgery alone. Ann Surg Oncol. 2008;15(11):3147-56.

9. American College of Surgeons [Internet]. National Cancer Database; [cited Feb 1]. Available from: https://www.facs.org/Quality-Programs/ Cancer/NCDB

10. Abel S, Hasan S, White R, Schumacher L, Finley G, Colonias A, et al. Stereotactic ablative radiotherapy (SABR) in early stage nonsmall lung cancer: comparing survival outcomes in adenocarcinoma and squamous cell carcinoma. Lung Cancer. 2019;128:127-33.

11. Lo H, Abel S, Finley G, Weksler B, Colonias A, Wegner R. Surgical resection versus stereotactic body radiation therapy in early stage bronchopulmonary large cell neuroendocrine carcinoma. Thorac Cancer. 2020;11(2):305-10.

12. National Cancer Data Base Participant User File (PUF) Data Dictionary [Internet]. Version PUF 16. American College of Surgeons. Available from: https://www.facs.org/-/media/files/ quality-programs/cancer/ncdb/puf_data_dictionary_2016.ashx

13. World Health Organization [Internet]. International classification of diseases for oncology. 2012 [cited 2020 Jan 28]. Available from: https://apps.who.int/iris/handle/10665/42344

14. Pichlmayr R, Weimann A, Klempnauer J, Oldhafer KJ, Maschek $\mathrm{H}$, Tusch G, et al. Surgical Treatment in proximal bile duct cancer: a single-center experience. Ann Surg. 1996;224(5):628-38.

15. Su CH, Tsay SH, Wu CC, Shyr YM, King KL, Lee CH, et al. Factors influencing postoperative morbidity, mortality, and survival after resection for hilar cholangiocarcinoma. Ann Surg. 1996;223(4):384-94.

16. Luvira V, Eurboonyanun C, Bhudhisawasdi V, Pugkhem A, Pairojkul C, Luvira V, et al. Patterns of Recurrence after Resection of Mass-Forming Type Intrahepatic Cholangiocarcinomas. 2016;17(10):4735-9.

17. Hu LS, Zhang XF, Weiss M, Popescu I, Marques HP, Aldrighetti $\mathrm{L}$, et al. Recurrence patterns and timing courses following curativeintent resection for intrahepatic cholangiocarcinoma. Ann Surg Oncol. 2019;26(80):2549-57.

18. Wang G, Wang Q, Fan X, et al. The significance of adjuvant therapy for extrahepatic cholangiocarcinoma after surgery. Cancer Manag Res. 2019;11:10871-82.

19. NIH U.S. National Library of Medicine [Internet]. Adjuvant chemotherapy with gemcitabine and cisplatin compared to standard of care after curative intent resection of biliary tract cancer (ACTICCA-1); 2020, Sep 23 [cited 2021 Feb 3]. Available from: https://www.clinicaltrials.gov/ct2/show/NCT02170090

20. Primrose JN, Fox RP, Palmer DH, Malik HZ, Prasad R, Mirza D, et al. Capecitabine compared with observation in resected biliary tract cancer (BILCAP): a randomised, controlled, multicentre, phase 3 study. Lancet Oncol. 2019;20(5):663-73.

21. Neoptolemos JP, Moore MJ, Cox TF, Valle JW, Palmer DH, McDonald $\mathrm{AC}$, et al. Effect of adjuvant chemotherapy with fluorouracil plus folinic acid or gemcitabine vs observation on survival in patients with resected periampullary adenocarcinoma: the ESPAC-3 periampullary cancer randomized trial. JAMA. 2012;308(2):147-56.

22. Valle J, Wasan H, Palmer D, Cunningham D, Anthoney A, Maraveyas A, et al. Cisplatin plus Gemcitabine versus Gemcitabine for Biliary Tract Cancer. NEJM. 2010;362:1273-81. 
23. Klinkenbijl JH, Jeekel J, Sahmoud T, Pel RV, Couvreur ML, Veenhof $\mathrm{CH}$, Arnaud JP, et al. Adjuvant radiotherapy and 5-fluorouracil after curative resection of cancer of the pancreas and periampullary region: phase III trial of the EORTC gastrointestinal tract cancer cooperative group. Ann Surg. 1999;230(6):776-82.

24. Lin YK, Hsieh MC, Wang WW, Lin YC, Chang WW, Chang CL, et al. Outcomes of adjuvant treatments for resectable intrahepatic cholangiocarcinoma: chemotherapy alone, sequential chemoradiotherapy or concurrent chemoradiotherapy. Radiother Oncol. 2018;128(3):575-83.

25. Hoehn RS, Wima K, Ertel A, Meier A, Ahmad S, Shah S, et al. Adjuvant chemotherapy and radiation therapy with improved survival for patients with extrahepatic cholangiocarcinoma. Ann Surg Oncol. 2015;22:1133-9.
26. Ecker BL, Vining CC, Roses RE, Maggino L, Lee MK, Drebin JA, et al. Identification of patients for adjuvant therapy after resection of carcinoma of the extrahepatic bile ducts: a propensity scorematched analysis. Ann Surg Oncol. 2017;24:3926-33.

27. Sur MD, Haejin I, Sharpe S, Baker MS, Weichselbaum RR, Talamonti MS, et al. Defining the benefit of adjuvant therapy following resection for intrahepatic cholangiocarcinoma. Ann Surg Oncol. 2015;22:2209-17.

Publisher's Note Springer Nature remains neutral with regard to jurisdictional claims in published maps and institutional affiliations. 